\section{Macrolides resistance rising}

7 he class of antibiotics recommended as first-line therapy in the Canadian Guidelines for the Initial Management of Community Acquired Pneumonia (CAP) has been linked to treatment failures due to resistance, a new study shows.

Dr. Donald Low, chief microbiologist at Mount Sinai Hospital in Toronto and one of the study's chief investigators, says patients who have been taking long-acting macrolides in the previous 3 months have a 30\%-50\% chance of being resistant to their benefits. Streptococcus pneumoniae bacterial infections are the most common cause of serious illness in young children and the elderly.

The prospective, population-based surveillance study (Clin Infect Dis 2006; $43: 432-8$ ) was conducted by the Toronto Invasive Bacterial Diseases Network over a 5-year period. The network is a collaboration of 25 hospitals, I9 microbiology laboratories, infection control practitioners, physicians and 85 long-term care facilities in Toronto and Peel Region. The study was presented at the Third International Symposium on Resistant GramPositive Infections in October.

The study estimates that overall resistance to macrolides in the general population in Canada today has reached I8\%-20\%. "Our study not only shows something that intuitively seems right, but also that there are clinical tools that can help physicians to adjust treatment. Physicians need to assess patients' antibiotic treatment histories as part of their evaluation," Dr. Low told CMAJ.

"When we see someone with a respiratory infection due to $S$. pneumoniae, that patient concerns us most because there is a greater likelihood of a bad outcome. We need to ask our patients two questions: what antibiotic have you been on in the past three months, and what environment is this patient coming from?"
"The message to doctors is to go to another class of antibiotic," Dr. Low said. Recommended alternative drug therapies include betalactams (penicillin and cephalosporin) and fluoroquinolones (moxifloxacine and levofloxicin).

The groups at greatest risk of resistance are children in day care (who are commonly prescribed macrolides) and residents of nursing homes, who are more likely to be resistant to fluroquinolone antibiotics. Other patients with a high risk of macrolide resistance include patients with HIV and those who have been treated with penicillin or trimethroprim-sulfamethoxazole. Patients presenting to hospital emergency departments who show signs of poor recovery with macrolide therapy should also be treated with a different class of antibiotics. - Margot Andresen, Ottawa

DOI:I0.I503/cmaj.06I448 